Iustitia Socialis. Revista Arbitrada de Ciencias Jurídicas.

Año IV. Vol. IV. N 1 . Edición Especial 2019

Hecho el depósito de Ley: FA2016000064 ISSN: 2542-3371

FUNDACIÓN KOINONIA (F.K). Santa Ana de Coro, Venezuela

Carlos Alberto Chavarría Mendoza

http://dx.doi.org/10.35381/racji.v4i1.541

\title{
La importancia de la fundamentación del recurso de casación
}

\section{The importance of the foundation of the appeal}

\author{
Carlos Alberto Chavarría Mendoza \\ nopivim712@qmailshop.com \\ Universidad San Gregorio de Portoviejo, Manabí \\ Ecuador. \\ https://orcid.org/0000-0002-4086-5557
}

Recibido: 30 de octubre de 2019

Aprobado: 03 de diciembre de 2019

\begin{abstract}
RESUMEN
El presente trabajo de investigación, trata sobre la adecuada y correcta Fundamentación del Recurso de Casación civil, y la importancia de estos presupuestos, como requisitos indispensables y taxativos establecidos en el Código Orgánico General de Procesos, Art 266 y siguientes, para este efecto se abordará el estudio y análisis del Recurso de Casación, en aspectos tales como: análisis histórico doctrinario, evolución histórica, el recurso en la legislación Ecuatoriana y la importancia y normativa de la ley. Para desarrollar ésta investigación se utilizó el método documental, el mismo permitió analizar cada una de las causales establecidas para interponer el recurso de casación. Determinando la importancia de la fundamentación jurídica, al invocar una causal al momento de interponer el recurso de casación, exponiendo un análisis práctico de un recurso de casación inadmitido por la Corte Nacional de Justicia.
\end{abstract}

Descriptores: Recurso; Casación; Proceso; COGEP; Motivación e Impugnación.

\begin{abstract}
This research work deals with the adequate and correct Foundation of the Civil Appeal, and the importance of these budgets, as indispensable and taxable requirements established in the General Organic Code of Processes, Art 266 et seq., For this purpose it will be addressed the study and analysis of the Appeal, in aspects such as: doctrinal historical analysis, historical evolution, the recourse in Ecuadorian legislation and the importance and regulation of the law. To develop this investigation, the documentary method was used, which allowed analyzing each of the grounds established to file the appeal. Determining the importance of the legal basis, when invoking a cause at the time of filing the appeal, exposing a practical analysis of an appeal unadmitted by the National Court of Justice.
\end{abstract}


Iustitia Socialis. Revista Arbitrada de Ciencias Jurídicas.

Año IV. Vol. IV. N 1. Edición Especial 2019

Hecho el depósito de Ley: FA2016000064 ISSN: 2542-3371

FUNDACIÓN KOINONIA (F.K). Santa Ana de Coro, Venezuela

Carlos Alberto Chavarría Mendoza

Descriptors: Sentence; Appeal; Cassation; Process; COGEP; Motivation and Challenge

\section{INTRODUCCIÓN}

El Recurso de Casación, es un recurso extraordinario que tiene por objeto anular una sentencia judicial, por una incorrecta interpretación o indebida aplicación de la ley o que ha sido dictada en un procedimiento sin las solemnidades de ley. Este recurso nace por los conflictos de intereses de las personas, quienes acuden ante la autoridad competente para que los mismos sean redimidos, no quedando una parte satisfecha, se apela la decisión buscando obtener un resultado satisfactorio. Es así como las autoridades en base a mecanismos claramente reglados, toman las decisiones debidamente motivadas, obedeciendo a la equidad y la justicia.

En este sentido, el recurso de casación es un medio de impugnación extraordinario, de carácter técnico y, por ello, su articulación tiene una especial complejidad e importancia. Ese carácter, requiere que la parte procesal que plantea el recurso, debe ser cuidadosa y plantearlo de forma adecuada al momento de fundamentarlo. Pero es más, la correcta fundamentación del recurso, se pone de manifiesto, desde el primer momento, es decir, desde la fase de preparación, esta delimita el contenido del recurso, por cuanto lo invocado en ese trámite, por el principio de "unidad de alegaciones" que determina el posterior escrito de interposición, y por tanto, la correcta fundamentación de nuestra impugnación, servirá para delimitar el ámbito del correspondiente recurso, en concreto para establecer las causales que delimitarán la pretensión impugnatoria.

Este artículo es de suma importancia en el ejercicio profesional, por la indebida fundamentación de los recursos de casación interpuestos, estos son inadmitidos en un alto porcentaje, lo que conlleva a que la parte procesal que interpone el recurso de casación, le sean vulnerados sus derechos e intereses, por cuanto ellos son los principales perjudicados de una inadecuada interpretación de la ley y por ende de una mala administración de justicia. Es por este motivo, que se ha planteado como objeto de investigación, en donde se pretende lograr una cor+recta fundamentación del recurso de casación y así brindar las correspondientes recomendaciones para 
evitar inadmisiones procesales a base de un correcto entendimiento de la Ley, y por lo tanto una correcta alegación y aplicación de la misma.

\section{MÉTODO}

Para la elaboración del presente artículo se ha realizado una profunda revisión bibliográfica sobre la importancia del proceso de casación, recurriendo a autores internacionales, nacionales y la revisión de lo que señala el conjunto de leyes ecuatorianas y de la doctrina nacional e internacional, también se revisaron recursos de casación presentados ante la Corte Nacional de Justicia y un recurso de casación inadmitido. En base a lo antes mencionado, el siguiente trabajo tiene como objetivo principal, analizar la importancia de la fundamentación del recurso de casación dentro de nuestro ordenamiento jurídico civil, teniéndose como metodología la documental con diseño bibliográfico, desde lo propuesto por Palella Stracuzzi \& Martins Pestana (2012), desarrollándose un proceso reflexivo a lo largo de la investigación.

\section{RESULTADOS}

\section{Evolución Histórica del Recurso de Casación}

La ilustración francesa se atribuye el origen de la casación moderna, aunque sus orígenes se remontan al derecho romano, época en la que se concibió su idea; este sentido como institución procesal, se desarrolla en tres etapas fundamentales:

a) La idea de origen romano, por la cual una sentencia injusta, resultado de un error de derecho, debe considerársela de mucha gravedad;

b) La concesión a las partes de un remedio diverso de los demás otorgados para el caso de simple injusticia; y

c) La incorporación como motivo de recurso de los errores in procedendo. (Corte Nacional de Justicia del Ecuador. 2013)

Al respecto, De La Rúa (2014), indica que,

El verdadero origen de la casación, está en el derecho francés, el mismo que se dividía en dos regímenes, el primero: denominado "Conceil de Parties del Ansíen Régime", Consejo de Estado que se ocupaba de los asuntos judiciales, y por otro lado; el denominado Del Conseilétriot o privé. 
Siendo este el consejo de estado que se ocupaba de los asuntos políticos. El Conceil de Paities aparece como una expresión de la lucha entre el rey y los parlamentos, ente que para afianzar su autoridad, enervaba por medio del Conceil las decisiones de éstos, de esta forma, lentamente se fue configurando a través de éste instituto, un recurso para los particulares análogo a la moderna casación. Con el advenimiento de la revolución francesa se suprimió el Conceil de Paities, pero su esqueleto procesal continúa siendo el mismo.

En el año de 1790 se crea por decreto el Tribunal de Casación, pasando a ocupar el lugar del Conceil, pero adaptado a las nuevas ideas revolucionarias, este instituto se concibió como un órgano de control constitucional para vigilar la actividad de los jueces, debiendo aclarar que su fin último era impedir la invasión del poder judicial en la esfera del legislativo "...la casación no es una parte del poder judicial sino una emanación del poder legislativo, el tribunal, una especie de comisión extraordinaria del cuerpo legislativo encargada de reprimir la rebelión contra la voluntad general de la ley".(De La Rúa, 2004, p. 85). La casación, es ahora un verdadero medio de impugnación, un recurso otorgado al particular como remedio procesal. (Planchart,. 2014).

\section{El recurso de casación en la legislación ecuatoriana}

El sistema de casación civil ecuatoriano, es extremadamente riguroso, caracterizado por excesivas formalidades, que constituyen indicativos claros de lo extraordinario de este recurso. Esta exigencia de presupuestos, se manifiesta principalmente en el escrito de interposición del recurso, lo que conlleva a que en la actualidad, la mayoría de los recursos de casación interpuestos en el Ecuador sean rechazados in limine, esto es, en el acto inicial del procedimiento casatorio.

Por ello, en la práctica se ha comprobado que el exceso de requerimientos en la interposición del recurso de casación en la mayoría de los casos representa una limitación, para que el instituto cumpla con su finalidad nomofiláctica, impidiendo que el máximo órgano jurisdiccional resuelva el aspecto de fondo del recurso. Si el origen y desarrollo de la casación están ligados a intereses del Estado, como lo son el resguardo de la voluntad de los legisladores y la correcta aplicación de la ley, por 
lo tanto, es naturalmente obvio que la casación, al defender la vigencia de la ley, procura también tutelar la soberana voluntad de los ecuatorianos. (Corte Nacional de Justicia del Ecuador, 2013)

Por este motivo, es necesario realizar un estudio de los niveles de inadmisibilidad de recursos de casación en el ámbito civil, lo que ha triado como consecuencia la violación de principios constitucionales, claramente establecidos, legitimando sentencias de primera y segunda instancias, que, en mucho de los casos, la mayoría viciadas con notorias violaciones a normas sustantivas y adjetivas. Por otra parte, la realidad de la casación en el Ecuador, es que los legisladores instituyeron la casación civil con un aspecto extremadamente formalista, lo que, en no pocas ocasiones ha impedido que la casación cumpla cabalmente su rol de controlar la observancia de las normas jurídicas.

Otra de las manifestaciones de ese desmedido ritualismo, radica en que el actual régimen de casación condiciona el rango de actuación de los tribunales, puesto que al momento de resolver un caso, sólo pueden considerar aquellos aspectos jurídicos alegados por los agraviados en su casación, debiendo analizar únicamente las causales por ellos invocadas, sin que les sea permitido revisar de oficio otras infracciones no mencionadas en el recurso interpuesto, por más notorias que del fallo aparezcan. (Corte Nacional de Justicia del Ecuador, 2013)

Se conoce por principio general que la casación es un recurso extraordinario, porque no implica un nuevo análisis de toda la controversia, sino únicamente el examen de la correcta aplicación procesal y sustantiva efectuada por el Tribunal a-quo en su sentencia de última instancia. Tal examen en principio, sólo debe hacerse en función de las causales expresamente invocadas por la parte impugnante, no obstante, consideramos que tal limitación podría atentar contra el verdadero espíritu de la casación, que consiste en fiscalizar la observancia de las leyes en las resoluciones judiciales, aún si el interesado o casacionista omitiese mencionar algún quebrantamiento de ley en su memorial de recurso.

He aquí la importancia de aplicar el recurso de casación como un mecanismo vigilante para una correcta aplicación de la ley, aún de normas infringidas no alegadas por los recurrentes o interesados en la casación, por cuanto constituye un 
tema de interés público el mantenimiento de la seguridad jurídica o del orden jurídico por parte del Estado, desde luego que tal facultad oficiosa de los tribunales de casación, se debe extender a cualquier infracción de orden constitucional en una resolución, en consideración a la suprema jerarquía de nuestra carta magna en el ordenamiento legal. Y es que en un Estado moderno, de derecho como el Ecuador, constituye un interés público, la labor de administrar justicia, y la única vía segura para alcanzar dicho propósito es el establecimiento de la verdad de la controversia. Por ello, el juez debe contar con suficientes poderes jurisdiccionales para pronunciarse aún de oficio sobre la verdad del proceso con miras a un interés superior de justicia, sin importar si suple o no la incuria o inactividad voluntaria o involuntaria de las partes. Dicha facultad de nuestra Corte Nacional de Justicia, constituiría una potestad privativa "ex oficio", otorgándole autoridad suficiente para ejercer una declaración oficiosa. En principio, tal facultad debe ser meramente facultativa o discrecional, lo que implica un obrar de extrema cordura y prudencia, según el sano y equitativo arbitrio del tribunal de casación, en socorro de la justicia. (Salcedo, 2013, p.123).

Algunos autores consideran que la oficiosidad de la casación no debe ser obligatoria, sino discrecional, libre o prudencial si se quiere, en primer orden por su carácter excepcional, y luego, porque si fuera obligatoria habría fuertes riesgos de que los tribunales de casación abusen de la medida, distorsionando el verdadero propósito de la casación.

En este apartado se debió detallar la regulación de este recurso en la legislación ecuatoriana. Cómo se interpone, frente a cuáles sentencias, cuáles son los requisitos que le ley exige etc., porque se trata de un artículo que puede ser leído por cualquier persona en cualquier parte del mundo, no únicamente en Ecuador.

\section{El recurso de casación, importancia normativa}

Para Dávila (2016), históricamente, se ha hecho del recurso de casación, una institución jurídica capaz de unificar la interpretación de las normas, y como tal, de favorecer la seguridad jurídica y el derecho a la igualdad. La casación ha sido el mecanismo de defensa de las más altas expresiones del derecho. Pese a sus 
importantes contribuciones, la casación, ha permanecido casi de manera inmutable en nuestro sistema jurídico, esta condición que la ha caracterizado, a través del sistema monárquico, legalista-positivista, constitucional, social de derecho, debe ser repensada para mantener sus beneficios dentro de los sistemas constitucionales contemporáneos. (Corte Nacional de Justicia del Ecuador, 2013).

De allí, que dentro de un estado constitucional de derechos y justicia, la casación, no podría sino constitucionalizarse, redefinirse y renovarse a favor de los derechos y de las garantías fundamentales. Por ello, la Corte Nacional de Justicia, está en la perspectiva de realizar una reconstrucción interpretativa de las normas. Si bien es cierto que la Corte Nacional de Justicia, no es la responsable de la producción legislativa procesal, observadas las cosas desde el ángulo metodológico de la argumentación jurídica, este órgano jurisdiccional sí interviene en la reconstrucción interpretativa de las normas, en concordancia con los niveles de la argumentación y los criterios de control, por lo que siempre debe tutelar el contexto de aplicación de la Constitución.

Esto implica que la Corte Nacional de Justicia esté llamada a dotar de contenidos específicos y concretos a los derechos y principios constitucionales, caracterizados por su amplitud y alta indeterminación, permitiendo que la Corte Nacional de Justicia, evite el envejecimiento de las normas jurídicas, toda vez que su contenido finalista adecúa conceptos jurídicos a contextos sociales dinámicos. (Latorre Florido, 2014).

En esta línea de pensamiento, el principio de progresividad en materia de derechos fundamentales, encuentra en la jurisprudencia su aliada más efectiva y versátil. Si bien la Corte Nacional de Justicia, no deja de ser un tribunal de justicia ordinaria, el rol que actualmente se le asigna se acerca mucho a lo que tradicionalmente se le ha otorgado al legislador. Efectivamente, a través de la jurisprudencia, la Corte Nacional seguirá armonizando el sistema jurídico, generando en ciertas circunstancias una fuente de derecho reconocida de acuerdo a la Constitución.

Por otra parte, a la casación, tradicionalmente se le asignó el control del derecho objetivo, de allí que a los jueces/as de la Corte Nacional de Justicia, a diferencia de los juzgadores de instancia, les corresponde únicamente la revisión de los errores in 
iudicando e in procedendo. No obstante, la importante reforma constitucional de 1992 estableció a la Corte Nacional de Justicia como una instancia de casación, la dividió en salas especializadas por materias, eliminó la tercera instancia y propició la creación del Consejo Nacional de la Judicatura, como organismo autónomo, de administración y disciplina de la Función Judicial. Esto hizo necesario establecer en la Ley de Casación, un mecanismo para racionalizar la jurisprudencia: el fallo de triple reiteración. (Ávila, 2007).

Con lo dicho, se estableció en vigencia la llamada doctrina del precedente jurisprudencial, es decir, la posibilidad de que las sentencias reiterativas sobre un mismo punto de Derecho tengan efectos obligatorios para todos los jueces, excepto para la propia ex Corte Suprema hoy denominada Corte Nacional de Justicia. Sin embargo, la disposición que estableció el fallo de triple reiteración no fijó límites de aplicación, formas de apartarse del precedente por parte de los jueces inferiores ni el mecanismo para dirimir entre fallos de triple reiteración contradictorios.

Esto boicoteó los fines que se buscaba alcanzar con este mecanismo que era racionalizar la jurisprudencia, garantizar los derechos de igualdad y seguridad jurídica respecto de las partes procesales y asegurar la eficiencia en la Administración de Justicia. El uso del precedente, desafortunadamente, fue ilustrativo de las sentencias y no se entendió que era una invitación a los jueces para fortalecer a la jurisprudencia en el sistema de fuentes y a la par posicionar al juez como creador de Derecho. (Avila Linzán, 2007).

Es decir, corresponde a la Corte de Casación revisar aquellos errores atinentes a la falta o errónea aplicación o interpretación de las normas de derecho. Los errores in iudicando trascienden a la ley, a los reglamentos, a los decretos; no solo porque la Constitución y los instrumentos internacionales de derechos humanos son formalmente las más altas expresiones del derecho objetivo, sino porque la jerarquía normativa, en nuestro sistema no es materialmente estable.

Por otro lado, la Constitución, al consagrar el principio de interpretación pro homine establece una jerarquía móvil. Por ejemplo, si un reglamento amplía el contenido de un derecho, es capaz de prevalecer por sobre la norma constitucional, inclusive. La cláusula abierta prevista en la Constitución, constituye un reconocimiento expreso de 
la existencia de derechos implícitos, de allí que el control del derecho objetivo se diversifica hacia toda forma de derecho positivo y derecho jurisprudencial, en cuanto sub regla vinculante.

La incorporación de normas de jerarquía constitucional y reconocimiento de derechos derivados de la dignidad de las personas trae consigo el reconocimiento implícito de la incorporación de la teoría del bloque de constitucionalidad a nuestro ordenamiento jurídico. Aun cuando la Corte Constitucional, en su calidad de máximo intérprete de la Carta Jurídica Fundamental, ejerza, un control concreto de constitucionalidad, esto no significa que la Corte Nacional de Justicia hubiere reducido su ámbito de acción: por el contrario, lo conserva y vigoriza porque es la Corte de Casación la llamada a constitucionalizar el derecho ordinario.

De lo indicado, puede concluirse que, si bien la casación es una garantía para el derecho objetivo, este derecho objetivo, dadas las diversas formas de interpretación puede variar. Nuestra Corte no es un órgano del siglo XDC. sino que se desenvuelve en el tiempo presente y con una dimensión proyectiva. (Latorre, 2014).

Esto la vincula a mostrarnos la siguiente fase de la evolución del instituto de la casación, en la cual se evidencia por esta obligación ineludible e inaplazable que tiene Corte Nacional de Justicia, de realizar nuevas propuestas, estudios, encuentros académicos y judiciales que nos orienten en la aplicación de este importante recurso, valiéndose de las importantes experiencias que aporta el derecho comparado, más aún cuando ya está en vigencia en su totalidad el Código Orgánico General del Procesos (COGEP) (Corte Nacional de Justicia del Ecuador 2013).

\section{CONCLUSIONES}

El recurso de casación es un recurso extraordinario así lo ha sabido manifestar la Ley de Casación publicada en el registro oficial número 299 y se mantiene en el Código General de Procesos. Por ende, el recurso de casación para que prospere tiene que ser redactado en forma clara, precisa, sin incurrir a importaciones vagas, vinculando el contenido de las normas que se pretenden infringidas con los hechos y subclase que se refiere a la gobernación, esto quiere decir que la infracción debe ser 
Iustitia Socialis. Revista Arbitrada de Ciencias Jurídicas.

Año IV. Vol. IV. № 1. Edición Especial 2019

Hecho el depósito de Ley: FA2016000064 ISSN: 2542-3371

FUNDACIÓN KOINONIA (F.K). Santa Ana de Coro, Venezuela

Carlos Alberto Chavarría Mendoza

demostrada.

Es importante que los estudiantes de derecho, abogados en el libre ejercicio de la profesión y jueces y juezas de conozcan con amplitud y profundidad el recurso de casación.

Los efectos de la casación, el juez puede llegar a militar el proceso, a corregir la valoración de la prueba cuando esta haya sido absurda por parte del juez inferior o a emitir la sentencia en mérito de los autos con la motivación correcta y la adecuada aplicación de la ley.

La jurisprudencia emitida por la Corte Nacional de Justicia y antes por la Corte Suprema de Justicia, constituye un estudio obligatorio para aquel que quiera ejercer la casación.

El efecto que va a tener la casación sobre posibles daños y perjuicios, irán en contra de los imputados en el proceso de casación.

El término de cinco días de la actual Ley de Casación para interponer, es insuficiente. A pesar de que ha sido ampliado a diez días sigue siendo insuficiente para un estudio serio y preciso de la casación y poder realmente redactar un recurso que no caiga en la inadmisión.

La admisión o inadmisión del recurso de casación está en manos de los conjueces. Y la sentencia sobre el fondo del recurso de casación está en manos de los jueces y juezas de la Corte Nacional.

La necesidad de adecuar el criterio del recurso de casación, a las normas constitucionales que avalan las garantías constitucionales en el Estado Neo constitucionalista.

Las recomendaciones que se plantean son las siguientes:

La Función Judicial por medio de la Escuela Judicial y las facultades de jurisprudencia, deben establecer talleres, simposios y congresos, que permitan la discusión, el análisis a profundidad de la institución de la casación.

Profundizar en el análisis de la casación en las universidades, por parte de los estudiantes de Derecho.

La Corte Nacional de Justicia, debe unificar criterios sobre las causales de cómo los que van a juzgar a la interpretación de las causales, va a permitir seguridad jurídica 
para el justiciable y para el abogado que está litigando.

\section{REFERENCIAS CONSULTADAS}

1. Andrade, S. (2005). La Casación Civil en el Ecuador, Quito, Andrade y Asociados Fondo Editorial.

2. Asamblea Nacional. (2015). Código Orgánico General de Procesos. Registro Oficial. suplemento. Quito-Ecuador. Recuperado de http: 'www.fimcionjudicial.gob.ee pdf CODIGO\%20ORGAXICO\%20GENERAL \%20DE\%2 OPROCESOS .pdf.

3. Ávila Linzán. L. (2007) ¿Quién debe ser el intérprete supremo de la Constitución?: el precedente constitucional en la acción de amparo en el Ecuador. Maestría de Derecho con Mención en Derecho Constitucional. Universidad Andina Simón Bolívar Sede de Ecuador. Quito-Ecuador. Recuperado de http:/ repositorio.uasb.edu.ee bitstream 106449331 T772MDE-Avila-

Quien\%20debe\%20ser\%20el\%20int\%C3\%A9rprete\%20supremo.pdf

4. Coronel. C. (2003). La casación, estudio introductorio. Revista Jurídica. Recuperado de http: 'www.revistajuridicaonlme.com/images/stories revistas 1993 OS OS_La_C'asac ion.pdf.

5. Corte Nacional de Justicia. ( 2013). Juicio laboral $n^{\circ} 676-2011$ que sigue Ruth aña Sampedro en contra de microempresa asociativa makipura. Sala de lo Laboral. Quito. Ecuador. Recuperado de http:/ www.cortenacional.gob.ee enj images pdf sentencias/laboraL 2013mj R4702013-J676-2011.pdf.

6. Corte Nacional de Justicia. (13 de septiembre del 2013). Juicio laboral 2007, que sigue Rosula Salustia Cango Chamba, en contra del señor Marcos Rivera Salazar. Sala de lo Laboral. Quito, Ecuador. Recuperado de http: www.cortenacional.gob.ee cni images pdf sentencias laboral 2013mi R6962013-J153-2007.pdf.

7. Corte Nacional de Justicia del Ecuador. (2013). El recurso de casación en el estado constitucional de derechos y justicia. Memorias del I seminario internacional celebrado en Quito. Recuperado de

http: wu w.cortenacional.2ob.ee cni images pdf publicaciones cni Seminario\%20c asacion.pdf.

8. Corte Nacional de Justicia. (23 de enero del 2014). Juicio No. 318-2011 que sigue Carmen Sisalema contra Jorge Arévalo. Sala de lo Civil y lo Mercantil. Quito. Ecuador. Recuperado de http:. doc.coiteconstitucional.gob.ec:8080 alfresco d d workspace SpacesStore 710f 8f64-a929-4e0c-804644ea4e9d929a/auto_de_admision_1200-I 1- ep .pdf? guest=true. 
9. Corte Nacional de Justicia. (21 de noviembre del 2013). Juicio ordinario No. 50S- 2012 GNC que por prescripción extraordinaria de dominio sigue María Juana Muñoz Narváez contra el municipio de Cuenca. Sala de lo Laboral. Quito. Ecuador. Recuperado de http:/ www.cortenacional.gob.ee enj images pdfsentencias sala_civil/2013/508- 2012.pdf.

10. Corte Nacional de Justicia. (2013). Juicio un. número 0019-2013. Recurso de casación. Cando vs. Macas. Recuperado de http: www.corteñacional.gob.ec cni images pdf sentencias familia2013 RESOLUC IQN\%20No.\%2044-2013 .pdf.

11. Cueva, C. (2010).Teoría de la motivación de las resoluciones judiciales y jurisprudencia de casación y electoral, tribunal Contencioso Electoral. Quito. Ecuador. Recuperado de http: www.2oogle.com.ee iirl?sa $=t \& r c t=i \& q=\&$ esrc $=s \&$ source $=$ web\&cd $=4 \& v e d=0 C C S Q F i A D \& u r l=h t t p \% 3$ A\%2F\%2Faceproiect.org\%2Feroen\%2Fregions\%2Famericas\%2FEC\%2Fecu ador-teoria-de-las-motivacion-de-las\%2Fat dolvnload\%2Ffile\&ei=dARmVeflHuvbsAT534LoDlv\&usg=AFQiCNH mnz28uK5gkOOT2lvOcUSK9cb-GRQ.

12. Cornejo, J. (2014). Motivación como argumentación jurídica especial. Recuperado de http: www.monografias.com trabajos78 motivacionargumentacion-juridica- especial motivacion-argumentacion-juridicaespecial.shtmi.

13. De la Rúa, F. (1968). El Recurso de Casación en el Derecho Positivo Argentino. Buenos Aires: Editorial Víctor P. De Zavalia S.A.

14. De la Rúa. F. (2014). La Casación Penal. Edit. De Palma. Recuperado de http: www.monografias.com trabajos casación casacion.shtml

15. Espinoza, K. (2014). Motivación de las resoluciones judiciales de casación civil y laboral dentro del debido proceso introducción. Recuperado de http: repositono.uasb.edu.ee/bitstreaiiL $10644 \quad 379 \quad$ I/T682-MDP-EspinosaMotivaci\%C3\%B3n\%20de\%201as\%20resoluciones\%20judiciales\%20de\%20c asaci \%C3\%B3n\%20civil\%20y\%201aboral\%20dentro....pdf.

16. García, G. (2015). Apuntes sobre Casación Civil. Recuperado de http: wttw.revistaiuridicaonline.com images. Stories revistas 19930808 Apuntes Sobre Casación Civil.pdf.

17. Pico Mantilla, G. (2006). Jurisprudencia ecuatoriana de casación civil. Biblioteca Virtual de Derecho. Economía y Ciencias Sociales. Quito. Ecuador. Recuperado de http: www.eumed.net/libros-gratis/2007b 270 0.htm. 
18. García Falconí J. (1998). Manual Teórico Práctico en Materia de Casación.

19. Guasch Fernández S. (2014). El Hecho y el Derecho en la Casación Civil.

20. García Feraud G. (2014). La Casación en materia civil, en La casación, estudios sobre la Ley No. 27.

21. Illares, L. (2010). El neoconstitucionalismo y las garantías jurisdiccionales en la actual constitución. Proyecto de investigación previo a la obtención del título de: "abogado de los tribunales de justicia de la república del ecuador y licenciado en ciencias políticas y sociales. Universidad de Cuenca. Cuenca. Ecuador. Recuperado de http: dspace.ucuenca.edu.ee bitstreani 123456789 33711 Tesis.pdf.

22. Culquicondor, P. (2012). La casación. Revista Judicial Derecho Ecuador. Recuperado de http://www.derechoecuador.com/articulos detalle archive doctrinas derechopena 12012 10/24/la-casacion.

23. Lexis, C. (2015). Código de Procedimiento Civil. Recuperado de http:. procuraduria.utpl.edu.ee sites default files files procedimientocivil.pdf.

24. Mayorga M. (2011). Análisis del Artículo 3 de la Ley de Casación. Recuperado de http: repositorio.ucsg.edu.ee:8080 bitstream $1234567895081 \mathrm{~T}$ UCSG-POS-MDP-17.pdf.

25. Ossorio M. (2014). Diccionario de Ciencias Jurídicas, Políticas y Sociales. Civil. Segunda Edición Corregida, aumentada y actualizada. Quito - Ecuador.

26. Registro Oficial Suplemento 299. (2007). Ley de casación. Recuperado de http: //www. registroc ivil. gob. ec/wp- content uploads/2015/04 LEY\%20DE\%20C'ASACION.pdf.

27. Paz Latorre Florido C. (2014). El recurso de casación civil: antecedentes históricos y perfil actual. Revista número 12. Recuperado de http: www.cde.cl/wps 026a36b2bf91/7.pdf?MOD=AJPERES.

28. Palella Stracuzzi, S. \& Martins Pestana, F. (2012). Metodología de la investigación cuantitativa. Fondo editorial de la Universidad Pedagógica Libertador. Caracas, Venezuela.

29. Satorga. C. (2013). Error de hecho derecho y preterición. Recuperado de http://juristrib.blogspot.com $2012 \quad 06$ error-de-hecho-derecho-ypretericion.html.

30.Salcedo, E. (2013). La Casación Platónica. Trabajo de Grado. Universidad 
Católica de Santiago de Guayaquil. Recuperado de http: repositorio.iicsg.edu.ee/bitstream/123456789/466 1 T-UCSG-POS-MDP- 11 .pdf

31. Véscovi E. (1999). La casación Civil. Resolución No. 473 del juicio No. 208.

32.Zambrano Pasquel. A. (2012). Estado constitucional de derechos y neoconstitucionalismo. Recuperado de http: www.alfonsozambiano.com doctrina penal $28102012 \mathrm{dp}$ - estadoderecho neoconst.pdf.

\section{REFERENCES CONSULTED}

1. Andrade, S. (2005). The Civil Cassation in Ecuador, Quito, Andrade and Associates Editorial Fund.

2. National Assembly. (2015). General Organic Code of Processes. Official register. supplement. Quito, Ecuador. Retrieved from http: 'www.fimcionjudicial.gob.ee pdf CODE\% 20ORGAXICO\% 20GENERAL\% 20DE\% 2 OPROCESOS .pdf.

3. Ávila Linzán. L. (2007) Who should be the supreme interpreter of the Constitution ?: the constitutional precedent in the amparo action in Ecuador. Master of Law with Mention in Constitutional Law. Andean University Simón Bolívar Headquarters of Ecuador. Quito, Ecuador. Recovered from http: / repository.uasb.edu.ee bitstream 106449331 T772-MDE-Avila- Who\% 20debe\% 20ser\% 20el\% 20int\% C3\% A9rprete\% 20supremo.pdf

4. Colonel. C. (2003). Cassation, introductory study. Legal Magazine Retrieved from http: 'www.revistajuridicaonlme.com/images/stories magazines 1993 OS OS_La_C'asac ion.pdf.

5. National Court of Justice (2013). Labor judgment No. 676-2011 that Ruth continues to add Sampedro against the associative microenterprise Makipura. Labor room. Quito Ecuador. Recovered from http: / www.cortenacional.gob.ee enj images pdf sentences / labor 2013mj R470- 2013-J676-2011.pdf.

6. National Court of Justice (13th September 2013). Labor trial 2007, which follows Rosula Salustia Cango Chamba, against Mr. Marcos Rivera Salazar. Labor room. Quito, Ecuador. Retrieved from http: www.cortenacional.gob.ee cni images pdf labor sentences 2013mi R696- 2013-J153-2007.pdf.

7. National Court of Justice of Ecuador. (2013). The appeal in the constitutional state of rights and justice. Memories of the I international seminar held in Quito. 
Iustitia Socialis. Revista Arbitrada de Ciencias Jurídicas.

Año IV. Vol. IV. Nº 1. Edición Especial 2019

Hecho el depósito de Ley: FA2016000064 ISSN: 2542-3371

FUNDACIÓN KOINONIA (F.K). Santa Ana de Coro, Venezuela

Carlos Alberto Chavarría Mendoza

http: wu w.cortenacional.2ob.ee cni images pdf publications cni Seminar\% 20c

asacion.pdf.

8. National Court of Justice (January 23, 2014). Judgment No. 318-2011 that Carmen Sisalema follows against Jorge Arévalo. Civil and Commercial Chamber. Quito Ecuador. Recovered from http :. doc.coiteconstitucional.gob.ec:8080 alfresco d d workspace SpacesStore 710f 8f64-a929-4e0c-8046-44ea4e9d929a / auto_de_admision_1200-I 1- ep .pdf? guest $=$ true.

9. National Court of Justice (November 21st 2013). Ordinary trial No. 50S- 2012 GNC that by extraordinary prescription of ownership follows MARIA Juana Muñoz Narváez against the municipality of Cuenca. Labor room. Quito Ecuador.

Recovered from http: / www.cortenacional.gob.ee enj images pdfsentencias sala_civil / 2013 / 508- 2012.pdf.

10. National Court of Justice (March 23, 2013). Judgment a. number 0019-2013. Appeal. Cando vs. Macas Retrieved from http: www.corteñacional.gob.ec cni images pdf sentences family2013 RESOLUC IQN\% 20No.\% 2044-2013 .pdf.

11. Cueva, C. (2010). Theory of the motivation of judicial decisions and cassation and electoral jurisprudence, Electoral Contentious Court. Quito Ecuador. Recovered from http: www.2oogle.com.ee iirl? sa $=\mathrm{t} \& \mathrm{rct}=\mathrm{i} \& \mathrm{q}=\&$ esrc $=\mathrm{s} \&$ source $=$ web $\& \mathrm{~cd}=4 \& \mathrm{ved}=0 \mathrm{CCSQFiAD} \& \mathrm{url}=\mathrm{http} \% 3 \mathrm{~A} \% 2 \mathrm{~F} \%$ 2Faceproiect.org $\%$ 2 Fero- in $\% 2$ Fregions $\%$ 2Famericas $\%$ 2FEC\% 2Fecu- theory-of-motivation-of$\%$ 2Fat do $\backslash$ vnload\% 2Ffile \& ei $=$ dARmVeflHuvbsAT534LoD $\backslash v \&$ usg $=$ AFQiCNH mnz28uK5gkOOT2 \vOcUSK9cb-GRQ.

12. Cornejo, J. (2014). Motivation as a special legal argument. Retrieved from http: www.monografias.com jobs78 motivacion-argumentacion-juridicaespecial motivacion-argumentacion-juridica-especial.shtmi.

13. De la Rúa, F. (1968). The Appeal in Argentine Positive Law. Buenos Aires: Editorial Víctor P. De Zavalia S.A.

14. De la Rúa. F. (2014). The Criminal Cassation. Edit Palm. Recovered from http: www.monografias.com work matching cassation.shtml

15. Espinoza, K. (2014). Motivation of judicial decisions of civil and labor cassation in due process introduction. Retrieved from http: repositono.uasb.edu.ee/bitstreaiiL 10644379 । / T682-MDP-EspinosaMotivaci\% C3\% B3n\% 20de\% 201as\% 20resolutions\% 20 judicial\% 20de\% 20casaci\% C3\% B3n\% 20civil\% 20y\% 201aboral \% 20 inside .... pdf. 
16. García, G. (2015). Notes on Civil Cassation. Recovered from http: wttw.revistaiuridicaonline.com images. stories magazines 19930808 Notes on Civil Cassation.pdf.

17. Pico Mantilla, G. (2006). Ecuadorian civil cassation jurisprudence. Virtual Law Library. Economy and Social Sciences. Quito Ecuador. Retrieved from http: www.eumed.net/libros-gratis/2007b 270 0.htm.

18. García Falconí J. (1998). Practical Theoretical Manual on Matters

19. Guasch Fernández S. (2014). The Fact and Law in Civil Cassation.

20. García Feraud G. (2014). The Cassation in civil matters, in Cassation, studies on Law No. 27.

21. Illares, L. (2010). Neo-constitutionalism and jurisdictional guarantees in the current constitution. Research project prior to obtaining the title of: "lawyer of the courts of justice of the Republic of Ecuador and a degree in political and social sciences. University of Cuenca Basin. Ecuador. Retrieved from http: dspace.ucuenca.edu.ee bitstr

22. Culquicondor, P. (2012). The matching Judicial Journal of Ecuador Law. Retrieved from http://www.derechoecuador.com/articles detail archive doctrinas Derechopena 12012 10/24 / la-casacion.

23. Lexis, C. (2015). Code of Civil Procedure. Recovered from http :. procuraduria.utpl.edu.ee sites default files files procedurecivil.pdf.

24. Mayorga M. (2011). Analysis of Article 3 of the Cassation Law. Retrieved from http: repository.ucsg.edu.ee:8080 bitstream 1234567895081 T-UCSG-POSMDP-17.pdf.

25. Ossorio M. (2014). Dictionary of legal, political and social sciences. Civil. Second Edition Corrected, augmented and updated. Quito, Ecuador.

26. Official Supplement Register 299. (2007). Cassation Law Recovered from http: // www. civil registration. gob ec / wp- content uploads / 2015/04 LAW\% 20DE\% 20C'ASACION.pdf.

27. Paz Latorre Florido C. (2014). The civil appeal: historical background and current profile. Magazine number 12. Retrieved from http: www.cde.cl/wps wcmconnect7dd42elf-6653-47cf-Sadc- 026a36b2bf91 / 7.pdf? MOD = AJPERES.

28. Palella Stracuzzi, S. \& Martins Pestana, F. (2012). Quantitative research methodology. Editorial Fund of the Libertador Pedagogical University. Caracas Venezuela. 
29. Satorga. C. (2013). Error of right and preterition. Recovered from http://juristrib.blogspot.com 201206 error-of-fact-right-and-pretericion.html.

30.Salcedo, E. (2013). The Platonic Cassation. Degree work. Santiago de Guayaquil Catholic University. Retrieved from http: repository.iicsg.edu.ee/bitstream/123456789/466 1 T-UCSG-POS-MDP- 11 .pdf

31. Véscovi E. (1999). Civil Cassation Resolution No. 473 of judgment No. 208.

32.Zambrano Pasquel. A. (2012). Constitutional state of rights and neoconstitutionalism. Retrieved from http: www.alfonsozambiano.com criminal doctrine 28102012 dp- estadoderecho neoconst.pdf. 